\title{
RINGS WITH SUBEXPONENTIAL GROWTH AND IRREDUCIBLE REPRESENTATIONS
}

\author{
RONALD S. IRVING
}

\begin{abstract}
Some monoids and algebras are constructed with growth which is subexponential but not polynomially bounded. The algebras have homomorphic images which are primitive with polynomially bounded growth, but which do not satisfy a Nullstellensatz-type of property.
\end{abstract}

I. Introduction. Let $A$ be an algebra generated over a field $k$ by a finite set $X$. Let $A_{n}$ be the subspace of $A$ spanned by all words in $X$ of length less than or equal to $n$. We associate to $A$ the growth function

$$
g_{A}(n)=\operatorname{dim}_{k} A_{n} \text {. }
$$

Definition. (i) $A$ has exponential growth if $\lim g_{A}(n)^{1 / n}>1$. Otherwise, $A$ has subexponential growth.

(ii) $A$ has polynomially bounded growth if there exists a polynomial $p$ such that $g_{A}(n) \leqslant p(n)$ for all $n$.

As noted in [9], these definitions are independent of the generating set, and the limit in (i) always exists.

Let $M$ be a finitely generated monoid. Then a growth function can be associated to $M$ in a similar way, with $g_{M}(n)$ equal to the number of distinct words of length $\leqslant n$. Equivalently, the growth function is the one associated to the monoid ring $k[M]$, for any field $k$.

Work of Milnor, Wolf, Bass, and Guivarc'h has shown that a solvable group $G$ with subexponential growth is a finite extension of a nilpotnet group, and that such a group has polynomial growth [2], [3], [7], [10]. It is conjectured that any group with subexponential growth is nilpotent-by-finite, and so has polynomial growth. Recently, M. Smith proved that enveloping algebras of certain infinite-dimensional Lie algebras have subexponential growth which is not polynomially bounded [9].

In this paper, we construct a monoid and some algebras whose growth is subexponential but not polynomially bounded. The algebras are of additional interest because they do not satisfy a statement we call the Nullstellensatz:

Definition. An algebra $A$ over a field $k$ satisfies the Nullstellensatz if, for any irreducible $A$-module $V$, the division algebra $\operatorname{End}_{A} V$ is algebraic over $k$.

It is known that any finitely generated algebra over an uncountable field satisfies the Nullstellensatz. For arbitrary fields, any finitely generated PI-algebra satisfies the Nullstellensatz [1], as do group rings of polycyclic groups

Received by the editors December 21, 1977.

AMS (MOS) subject classifications (1970). Primary 16A20, 16A64.

๑ American Mathematical Society 1978 
[6] and enveloping algebras of finite-dimensional Lie algebras [8]. Note that these last two families are noetherian. Moreover, the previously constructed counterexamples have exponential growth (cf. [5]). This suggests that appropriate conditions which limit the "size" of an algebra over a field might imply the Nullstellensatz. But our examples show that subexponential growth is not such a condition. In fact, we construct a counterexample to the Nullstellensatz which has polynomially bounded growth. In addition, we have recently constructed a finitely generated noetherian algebra over any algebraic extension of a finite field which does not satisfy the Nullstellensatz.

\section{The monoid.}

THEOREM 1. Let $M$ be the monoid generated by $x$ and $y$, and satisfying the relations:

$$
x^{2}=x, \quad x y^{a} x y^{b} x=x y^{b} x y^{a} x, \text { for all } a, b>0 .
$$

Then $M$ has subexponential but not polynomially bounded growth.

Proof. The defining relations on $M$ imply that any word in $M$ can be written uniquely in the form:

$$
y^{a} x y^{i_{1}} x y^{i_{2}} x \cdots x y^{i_{m}} x y^{b} \text { or } y^{c}
$$

where $a, b, c$, and $m$ are greater than or equal to 0 , and $0<i_{1}<i_{2} \leqslant \cdots<$ $i_{m}$.

Let $p(n)$ be the standard partition function, and let $g=g_{M}$. Define $q(n)$ to be the number of choices of integers $0<i_{1}<\cdots<i_{m}$ such that

$$
1+i_{1}+1+\cdots+i_{m}+1=n
$$

for $n>1$, and set $q(0)=q(1)=1$. Then $q(n)$ is the same as the number of partitions of $n-1$ by integers larger than 1 , from which we see that

$$
q(n)=p(n-1)-p(n-2) \text {. }
$$

Let $\Delta g$ denote the first difference of the growth function $g$, so that $\Delta g(n)$ is the number of words of length $n$. Then

$$
\Delta g(n)=\sum_{i=0}^{n-1}(i+1) q(n-i)+1 .
$$

To obtain this formula, notice that for each fixed $i<n-1$, there are $i+1$ ways to choose $(a, b)$ so that $a+b=i$, and the remaining possibilities for a word of length $n$ correspond to the choices that are counted by $q(n-i)$.

We can now compute that the second difference is:

$$
\Delta^{2} g(n)=\Delta g(n)-\Delta g(n-1)=\sum_{i=1}^{n} q(i) .
$$

By (*), we deduce that $\Delta^{2} g(n)=p(n-1)$.

It is well known that the partition function has subexponential growth, but is not polynomially bounded. This follows from the famous asymptotic formula: 


$$
p(n)-e^{A \sqrt{n}} / 4 n \sqrt{3},
$$

where $A=\pi \sqrt{2 / 3}$. Since the first and second differences of $g$ are all nonnegative, $g(n) \geqslant \Delta^{2} g(n)$, so $g$ cannot be polynomially bounded. That $g$ is still subexponential follows from the following lemma:

LEMMA. $f(n)$ has subexponential growth if and only if $\Delta f(n)$ does.

For a short proof using generating functions, see [9].

III. The algebras. Our algebra construction will be somewhat different, so that we can construct the desired irreducible representation. Let $K$ be a field, and let $T=\left\{n_{1}<n_{2}<n_{3}<\ldots\right\}$ be a strictly increasing set of positive integers, not necessarily infinite. Let $A$ be the algebra generated over $K$ by $x$ and $y$, and satisfying the relations

$$
\begin{aligned}
x y^{a} x & =0, \quad a \notin T \text { and } a \geqslant 0, \\
x y^{a} x y^{b} x & =x y^{b} x y^{a} x, \quad a, b \in T .
\end{aligned}
$$

Notice in particular that $x^{2}=0$.

A $K$-basis for $A$ consists of monomials of the form

$$
y^{a} x y^{i_{1}} x \cdots x y^{i_{m}} x y^{b} \text { or } y^{c}
$$

where $a, b, c$, and $m$ are greater than or equal to 0 , and $0<i_{1} \leqslant i_{2} \leqslant \ldots<$ $i_{m}$, with each $i_{j} \in T$. If $T$ is taken to be the set of positive integers, then the growth function for $A$ is identical to the growth function for the monoid ring $K[M]$. So $A$ has subexponential but not polynomially bounded growth. More generally, we prove:

THEOREM 2. If $T$ is finite, of cardinality $r$, then $A$ has growth bounded above and below by polynomials of degree $r+2$. If $T$ is infinite, then $A$ has subexponential but not polynomially bounded growth.

Proof. Let $T^{\prime}$ be the set of integers $\left\{n_{j}+1: n_{j} \in T\right\}$. Let us associate to $T^{\prime}$ the restricted partition function $p\left(n, T^{\prime}\right)$, which counts the number of partitions of $n$, all of whose summands lie in $T^{\prime}$, and set $p\left(0, T^{\prime}\right)=1$. Then the first difference of the growth function $g$ is

$$
\Delta g(n)=g(n)-g(n-1)=\sum_{i=0}^{n-1}(i+1) p\left(n-i-1, T^{\prime}\right)+1
$$

One sees this just as in the proof of Theorem 1. It follows that the third difference is $\Delta^{3} g(n)=p\left(n-1, T^{\prime}\right)$.

For any choice of $T$, we have $p\left(n, T^{\prime}\right) \leqslant p(n)$, so $\Delta^{3} g$ has subexponential growth. Therefore $A$ has subexponential growth, by the lemma of Theorem 1 .

Assume that $T$ is infinite, and let $T_{r}=\left\{n_{1}, \ldots, n_{r}\right\}$. Then the algebras $A\left(T_{r}\right)$ are images of the algebra $A=A(T)$. As a result, if the first statement of the theorem is true, $A$ cannot have polynomially bounded growth.

We may assume then that $T=T_{r}$ for some $r$. The generating function for $p\left(n, T^{\prime}\right)$ is 


$$
F(t)=\prod_{i=1}^{r}\left(1-t^{n_{i}+1}\right)^{-1},
$$

and since $p\left(n, T^{\prime}\right)=\Delta^{3} g$, the generating function for $g(m)$ is $(1-t)^{-3} F(t)=$ $G(t)$. Let $n$ be the least common multiple of the $n_{i}+1$, and let $c_{1}$, $c_{2}, \ldots, c_{n_{1}}+\cdots+n_{r}$ be an enumeration of all the $\left(n_{i}+1\right)$ st roots of unity other than 1 , for $i=1, \ldots, r$. Then

$$
G(t)=1 /(1-t)^{r+3} \prod_{j}\left(1-c_{j} t\right) .
$$

The basic theorem for rational generating functions [4] implies that there are polynomials $P_{j}$, each of degree $\leqslant r-1$, and a polynomial $P$ of degree $r+2$ such that

$$
g(m)=\sum_{j} P_{j}(m) c_{j}^{m}+P(m)
$$

Hence for each $1 \leqslant k<n$, we find that on the set $\{i n+k\}, g$ acts as a polynomial of degree $r+2$. This proves the theorem.

IV. The representation. Let $R$ be any countably infinite domain, with field of fractions $K$, and let $V$ be the $K$-vector space with basis $v_{0}, v_{1}, \ldots$ We shall choose the set $T$ to be any infinite set satisfying the condition

$$
n_{i+1}-n_{i}>n_{i}-n_{i-1} \text { for all } i>1 \text {. }
$$

Let $A$ be the $K$-algebra associated to $T$, as in the previous section, and let $B$ be the subring of $A$ generated by $R, x$, and $y$.

We make $V$ into an $A$-module as follows:

$$
\begin{aligned}
& y \cdot v_{m}=v_{m+1}, \\
& x \cdot v_{m}=0 \text { if } m \notin T, \text { and } \\
& x \cdot v_{n_{i}}=a_{i} v_{0},
\end{aligned}
$$

where the $a_{i}$ are nonzero constants in $K$.

THEOREM 3. The vector space $V$ is an irreducible $A$-module. If the elements $a_{i}$ are chosen to enumerate $K-R$, then $V$ is irreducible as a $B$-module.

Proof. Let $v \in V$ be a nonzero vector. Then for some $i$, the vector $y^{i} v$ involves only one $v_{n_{j}}$ nontrivially, because of condition (*). The vector $x y^{i} v$ is a nonzero multiple of $v_{0}$, and it is now clear that $A \cdot v=V$. To see that $B \cdot v=V$, it suffices to show that $B \cdot v$ contains $K \cdot v_{0}$. We already know that $B \cdot v$ contains $a \cdot v_{0}$ for some $a \neq 0$ in $K$. The vector $x y^{n_{i}} \cdot a v_{0}=a a_{i} v_{0}$ and since the $a_{i}$ include all inverses of elements in $R$, we find that

$$
K \cdot v_{0} \subset B \cdot a v_{0} \subset B \cdot v,
$$

as desired.

In the representation above, no nonzero element of $R$ annihilates $V$, which shows that $B$ does not satisfy what we call the strong Nullstellensatz:

Definition. An $R$-algebra $A$ satisfies the strong Nullstellensatz if, for any irreducible $A$-module $M$, the prime ideal $R \cap$ ann $V$ is a maximal ideal. 
In particular, let $k$ be a countable field, and set $R=k[t]$. Then $B$ does not satisfy the Nullstellensatz as a $k$-algebra. We now claim that as a $k$-algebra, $B$ has subexponential but not polynomially bounded growth. In fact, $B$ has the same growth function as a $k$-algebra that $A[t]$ does as a $K$-algebra. The growth function of $A[t]$ has $g_{A}$ as its first difference, and so the claim follows.

Continuing with this example, let us assume that $R=k[t]$, with $k$ a countable algebraically closed field, and let the elements $a_{j}$ equal $\left(t-d_{j}\right)^{-1}$, for some enumeration $d_{j}$ of the elements of $k$. Then the associated module $V$ is still irreducible over $B$. Let $I$ be the annihilator of $V$.

THEOREM 4. The k-algebra B/I has polynomially bounded growth and does not satisfy the Nullstellensatz.

Proof. The Nullstellensatz does not hold because $I \cap k[t]=(0)$. So $t$ is a transcendental element in the endomorphism ring of $V$. It remains to show that $B / I$ has polynomially bounded growth. Observe that for any word $w=x y^{i_{1}} x \cdots x{ }^{i_{m}} x$ with the $i_{j}$ 's in $T$, and distinct, we have

$$
\begin{aligned}
w \cdot v_{s} & =a_{i_{1}} a_{i_{2}} \cdots a_{i_{m}} a_{t} \cdot v_{0} \quad \text { if } s=n_{t} \in T, \\
& =0 \quad \text { if } s \notin T .
\end{aligned}
$$

The element $a_{i_{1}} \cdots a_{i_{m}}=\Pi_{j=1}^{m}\left(t-d_{i,}\right)^{-1}$ can be written via partial fractions as $\sum_{j=1}^{m} b_{j} a_{i_{j}}$ for some elements $b_{j}$ in $k$. Then

$$
w \cdot v_{s}=\left(\sum_{j=1}^{m} b_{j} a_{i j}\right) a_{t} v_{0}
$$

which is the same as

$$
\left(\sum_{j=1}^{m} b_{j} x y^{i_{j}} x\right) \cdot v_{s}
$$

Therefore $I$ contains the element

$$
w-\sum_{j=1}^{m} b_{j} x y^{i_{j}} x
$$

A similar argument, using partial fractions, shows that in $B / I$, any word of the form $x y^{i_{1}} x \cdots x y^{i_{m}} x$ is equal to a $k[t]$-linear combination of words of the form $x y^{i} x y^{i}{ }^{i} x \cdots x y^{i} x$. This implies that as a $k$-vector space, $B / I$ is spanned by words

$$
\left\{t^{i} y^{a} x y^{r} x y^{r} x \cdots x y^{r} x y^{b}: i, a, b \geqslant 0 ; r \in T\right\} \cup\left\{t^{i} y^{a} x y^{b}\right\} \cup\left\{t^{i}{ }^{a}\right\} .
$$

We can eliminate more monomials, but it is unnecessary. For the above set has growth function bounded by a polynomial, and so $B / I$ has polynomially bounded growth.

\section{REFERENCES}

1. S. Amitsur and C. Procesi, Jacobson-rings and Hilbert algebras with polynomial identities, Ann. Mat. Pura Appl. 71 (1966), 61-71. 
2. H. Bass, The degree of polynomial growth of finitely generated nilpotent groups, Proc. London Math. Soc. 25 (1972), 603-614.

3. Y. Guivarc'h, Croissance polynomiale et périodes des fonctions harmoniques, Bull. Soc. Math. France 101 (1973), 333-379.

4. M. Hall, Combinatorial theory, Blaisdell, Waltham, Mass., 1967.

5. R. Irving, Some primitive group rings (to appear).

6. M. Lorenz, Primitive ideals of group algebras of supersoluble groups, Math. Ann. 225 (1977), 115-122.

7. J. Milnor, Growth of finitely generated solvable groups, J. Differential Geometry 2 (1968), 447-449.

8. D. Quillen, On the endomorphism ring of a simple module over an enveloping algebra, Proc. Amer. Math. Soc. 21 (1969), 171-172.

9. M. Smith, Universal enveloping algebras with subexponential but not polynomially bounded growth, Proc. Amer. Math. Soc. 60 (1976), 22-24.

10. J. Wolf, Growth of finitely generated solvable groups and curvature of Riemannian manifolds, J. Differential Geometry 2 (1968), 421-446.

Department of Mathematics, Brandeis University, Waltham, Massachusetts 02154 